\title{
Resonant absorption and the spectrum of 5-min oscillations of the Sun
}

\section{Influence of inhomogeneity of the canopy magnetic field on fine structure}

\author{
V. I. Zhukov^ \\ Central Astronomical Observatory of Russian Academy of Sciences, 65 Pulkovo, 196140 St. Petersburg, Russia
}

Received 25 September 2000 / Accepted 10 January 2001

\begin{abstract}
We studied influence of magnetic field on the spectrum of 5-min solar oscillations. The calculations were made within a two-layer shell model. The lower non-magnetized layer is characterized by a linear decrease in temperature with height while in the upper layer (with an inhomogeneous horizontal magnetic field) the temperature increases and reaches the coronal value at a height of about $2500 \mathrm{~km}$. The fine structure of the spectrum of 5 -min oscillations was calculated for $f_{-}, p_{1^{-}}$and $p_{2^{-}}$modes for large $k$.
\end{abstract}

Key words. Sun: oscillations - chromosphere - MHD

\section{Introduction}

Recently, considerable efforts have been made to study the influence of atmospheric magnetic field on the spectrum of 5-min oscillations. Originally, attempts to take into account the influence of the canopy magnetic field on the spectrum of 5-min oscillations were induced by the need to understand the reason for minor differencies between theoretically calculated and observed frequencies of the oscillations and also for minor variations in the frequencies of oscillations with the phase of a solar cycle (Campbell \& Roberts 1989; Evans \& Roberts 1990; Jain \& Roberts 1994a, 1994b).

Soon, however, it became clear that the canopy magnetic field contains Alfvén and cusp resonant levels for 5-min oscillations and hence the influence of the canopy magnetic field cannot be reduced to simple variation for eigenfrequencies of the oscillations. The presence of resonant levels results not only in some variation in frequencies of 5-min oscillations, but also in resonant heating of the upper atmosphere (Zhukov 1997a, 2000; Tirry et al. 1998; Pintér \& Goossens 1999; Vanlommel \& Goossens 1999) which makes it possible to consider the entire problem of nonradiative heating of the solar upper atmosphere from a new point of view (Zhukov 1992).

In this study, the fine structure of the spectrum of 5 -min oscillations is calculated taking into account their resonant absorption in the area of the canopy magnetic

^ e-mail: zhukov-spb@mail.ru field. As in the study by Zhukov (2000), the calculations were made for the two-layer model of the solar shell consisting of the lower layer without a magnetic field and with a linear decrease in temperature with height and the upper layer with a rapid increase in temperature, reaching the coronal value at a height of $\sim 2500 \mathrm{~km}$, but unlike previous papers (Zhukov 1997a, 2000), with an inhomogeneous horizontal magnetic field.

\section{The basic equations}

To accurately derive the frequencies of the 5-min oscillations, which could be suitable for comparison with observations, in the present paper, as in Zhukov (1997a, 2000), we calculate proper frequencies of a waveguide with

1. the bottom layer without a magnetic field; temperature falls with height linearly;

2. the top layer with the nonhomogeneous magnetic field and sharp increase of temperature up to the coronal value at a height about $2500 \mathrm{~km}$.

\subsection{The convective zone}

As is known, to describe adequately the 5 -min oscillations with sufficiently large $\ell$ to a first approximation, it is possible to restrict the consideration to the plane-layer model with a linear temperature change in the upper layers of the convective zone. 
In a Cartesian system of coordinates with the $Z$-axis directed upwards, for a layer $(z<0)$ with a linear profile of temperature, the basic equation for vertical components of velocity $v_{z}$ has the form (Nye \& Thomas 1976; Evans \& Roberts 1990)

$$
\begin{aligned}
c^{2}\left(\omega^{2}-\right. & \left.c^{2} k^{2}\right) \frac{\mathrm{d}^{2} v_{z}}{\mathrm{~d} z^{2}}+\left[c^{2} k^{2} \frac{\mathrm{d} c^{2}}{\mathrm{~d} z}-g \gamma\left(\omega^{2}-c^{2} k^{2}\right)\right] \frac{\mathrm{d} v_{z}}{\mathrm{~d} z} \\
+ & {\left[\left(\omega^{2}-c^{2} k^{2}\right)^{2}+\frac{(\gamma-1) g^{2} k^{2}}{\omega^{2}}\left(\omega^{2}-c^{2} k^{2}\right)\right.} \\
& \left.-g k^{4} c^{2} \frac{\mathrm{d} c^{2}}{\mathrm{~d} z}\right] v_{z}=0 .
\end{aligned}
$$

Pressure fluctuations in an acoustic wave are described by the equation

$p=i \frac{\rho_{0} \omega}{\omega^{2}-c^{2} k^{2}}\left(c^{2} \frac{\mathrm{d} v_{z}}{\mathrm{~d} z}-g v_{z}\right)$.

Henceforth we choose for all perturbed values the dependence from $x, y$ and $t$ in the form $\exp i\left(k_{x} x+k_{y} y+\omega t\right)$ and use the following notations: $\rho_{0}$ is the undisturbed density, $c$ the velocity of sound, $g$ the gravity acceleration which is considered constant, $\gamma$ the adiabatic index and $k^{2}=k_{x}^{2}+k_{y}^{2}$.

In general, the Eq. (1) is valid for any dependence of the sound velocity on height. In this study, however, as it was already noted, we shall restrict our consideration to the case when for $z<0$

$c^{2}=c_{01}^{2}\left(1-\frac{z}{z_{1}}\right)$.

\subsection{The chromosphere - corona}

We accept for the upper layer $(z>0)$ the dependence of the squared velocity of sound on $z$ in the form

$c^{2}=c_{02}^{2}\left(1+\delta\left[\tanh \left(\alpha\left(\frac{z}{z_{2}}-\beta\right)\right)-\tanh (-\alpha \beta)\right]\right)$.

We also assume that in this layer, the nonhomogeneous horizontal magnetic field $\boldsymbol{H}_{0}(z)$ exists. Then, in a Cartesian system of coordinates with $Z$-axis directed upwards and $X$-axis along the magnetic field, the basic equation for the vertical component of the velocity $v_{z}$ is

$\frac{\mathrm{d}}{\mathrm{d} z}\left[\rho_{0}\left(c^{2}+v_{\mathrm{A}}^{2}\right) \frac{\left(\omega^{2}-\omega_{\mathrm{A}}^{2}\right)\left(\omega^{2}-\omega_{\mathrm{T}}^{2}\right)}{\left(\omega^{2}-\omega_{+}^{2}\right)\left(\omega^{2}-\omega_{-}^{2}\right)} \frac{\mathrm{d} v_{z}}{\mathrm{~d} z}\right]$

$+\frac{\mathrm{d}}{\mathrm{d} z}\left[g \rho_{0}\left(1-\frac{\omega^{2}\left(\omega^{2}-\omega_{\mathrm{A}}^{2}\right)}{\left(\omega^{2}-\omega_{+}^{2}\right)\left(\omega^{2}-\omega_{-}^{2}\right)}\right)\right] v_{z}$

$+\rho_{0}\left(\omega^{2}-\omega_{\mathrm{A}}^{2}\right)\left[1-\frac{g^{2} k^{2}}{\left(\omega^{2}-\omega_{+}^{2}\right)\left(\omega^{2}-\omega_{-}^{2}\right)}\right] v_{z}$

$+\frac{\mathrm{d}}{\mathrm{d} z}\left[\frac{\left(\omega^{2}-\omega_{\mathrm{A}}^{2}\right)\left(\omega^{2}-c^{2} k_{x}^{2}\right)}{\left(\omega^{2}-\omega_{+}^{2}\right)\left(\omega^{2}-\omega_{-}^{2}\right)} \frac{\mathrm{d}}{\mathrm{d} z}\left(\frac{H_{0}^{2}}{8 \pi}\right) v_{z}\right]$

$-g \frac{\omega^{2} k_{y}}{\left(\omega^{2}-\omega_{+}^{2}\right)\left(\omega^{2}-\omega_{-}^{2}\right)} \frac{\mathrm{d}}{\mathrm{d} z}\left(\frac{H_{0}^{2}}{8 \pi}\right) v_{z}=0$.
Fluctuations of total pressure in a wave are given by the equation

$$
\begin{aligned}
& p+\frac{H_{0} h_{x}}{4 \pi}=\frac{i}{\omega} \frac{\left(\omega^{2}-\omega_{\mathrm{A}}^{2}\right)}{\left(\omega^{2}-\omega_{+}^{2}\right)\left(\omega^{2}-\omega_{-}^{2}\right)} \\
& \times\left[\rho_{0}\left(c^{2}+v_{\mathrm{A}}^{2}\right)\left(\omega^{2}-\omega_{\mathrm{T}}^{2}\right) \frac{\mathrm{d} v_{z}}{\mathrm{~d} z}-g \rho_{0} \omega^{2} v_{z}\right. \\
& \left.+\left(\omega^{2}-c^{2} k_{x}^{2}\right) \frac{\mathrm{d}}{\mathrm{d} z}\left(\frac{H_{0}^{2}}{8 \pi}\right) v_{z}\right],
\end{aligned}
$$

where

$\omega_{\mathrm{A}}^{2}=v_{\mathrm{A}}^{2} k_{x}^{2}, \omega_{\mathrm{T}}^{2}=c_{\mathrm{T}}^{2} k_{x}^{2}$,

and $v_{\mathrm{A}}^{2}=H_{0}^{2} / 4 \pi \rho_{0}, c_{\mathrm{T}}^{2}=c^{2} v_{\mathrm{A}}^{2} /\left(c^{2}+v_{\mathrm{A}}^{2}\right)$ and $\omega_{ \pm}^{2}$ are the roots of the following equation

$\omega^{4}-\omega^{2}\left(c^{2}+v_{\mathrm{A}}^{2}\right) k^{2}+c^{2} v_{\mathrm{A}}^{2} k^{2} k_{x}^{2}=0$

and $h_{x}$ is the perturbation of the $x$-component of the magnetic field in the wave. The other notations are the same as above.

It is easily to see that Eq. (3) has two types of singular points. The singular points $\omega^{2}=\omega_{\mathrm{A}}^{2}$ are known as Alfvén resonance points while the points $\omega^{2}=\omega_{\mathrm{T}}^{2}$ are the cusp resonance points.

Studies of wave propagation in a non-uniform medium with a magnetic field indicated that in the vicinity of the resonant levels an effective absorption of the waves occurs, and the amount of energy absorbed on a resonant level does not depend on the mechanism of wave dissipation (Joule dissipation, attenuation due to viscosity or radiative exchange) and on the values of the dissipation coefficients (provided that they are sufficiently small) (Goossens 1991).

Since Alfvén and cusp resonances for 5-min oscillations are located in a region of the canopy magnetic field, it is natural that the absorption of energy of 5-min oscillations on Alfvén and cusp resonances should result on the one hand in upper atmosphere heating, and on the other hand in a variation of the eigenfrequencies of 5 -min oscillations.

\subsection{The dispersion relation}

To calculate the spectrum of the acoustic waves captured in the shell we need the dispersion relation for the frequency of the captured wave $\omega$. This frequency (due to the tunnel leakage of wave energy into Alfvén and cusp resonance regions) is generally complex $\left(\omega=\omega_{r}+i \omega_{i}\right)$ (Zhukov 1997b).

The dispersion relation is derived from the boundary conditions (continuity of the vertical component of velocity and total pressure) at $z=0$

$v_{z 1}=v_{z 2}$,

$\frac{\partial p_{1}}{\partial t}-g \rho_{01} v_{z 1}=\frac{\partial}{\partial t}\left(p_{2}+\frac{H_{0} h_{x}}{4 \pi}\right)-g \rho_{02} v_{z 2}$, 


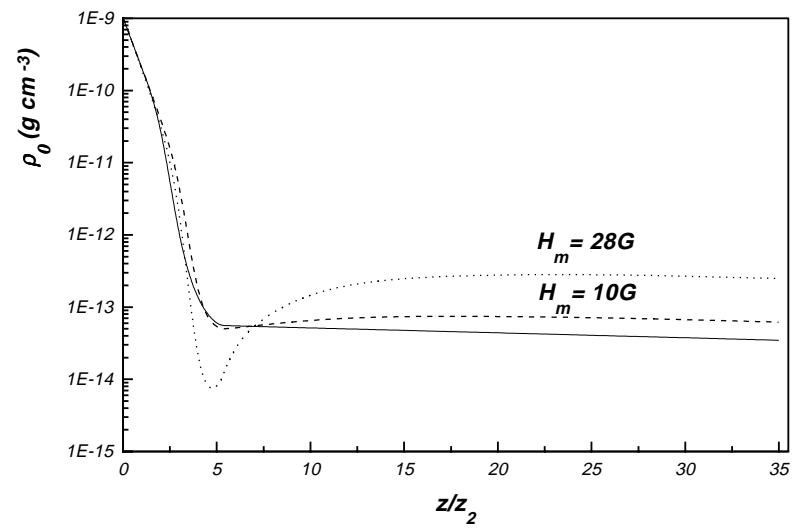

Fig. 1. Variation of density with height in the chromospherecorona for $z_{\mathrm{H}}=2000 \mathrm{~km}$

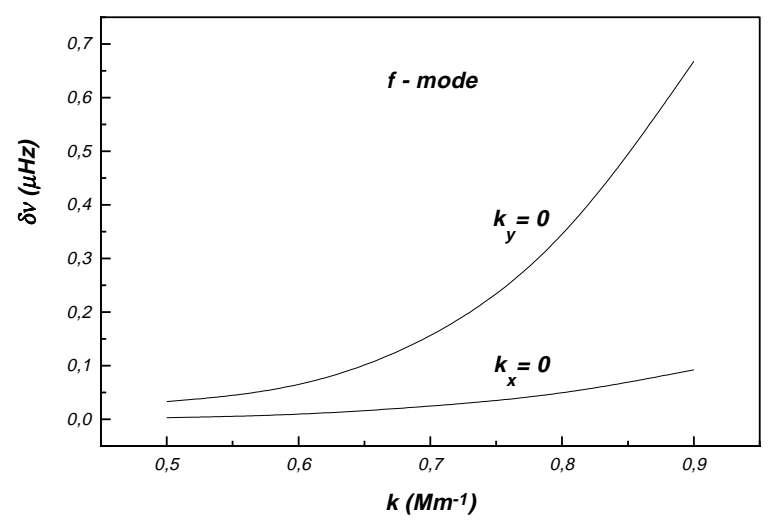

Fig. 2. Shift of the frequencies due to the resonant absorption for $f$-mode

and from the condition that the sum of kinetic and magnetic energy density of a wave approaches zero at $|z| \rightarrow \infty$.

The explicit form of the dispersion relation is given in the Appendix.

\section{Model parameters}

The dispersion relation was solved numerically with the following parameters of the convective zone:

$c_{01}=6.6829 \mathrm{~km} \mathrm{~s}^{-1}, g=274 \mathrm{~m} \mathrm{~s}^{-1}, \gamma=5 / 3, z_{1}=500 \mathrm{~km}$.

We adopt that there is a temperature and density jump at the boundary $(z=0)$ (Zhukov 2000):

$c_{02}^{2}=c_{01}^{2} \Delta$.

For the top layer (chromosphere-corona) it was accepted

$\alpha=3.0, \beta=4.0, \delta=50, \Delta=3.2, z_{2}=500 \mathrm{~km}$.

In this study we consider the canopy magnetic field inhomogeneous. Generally speaking, the structure of the canopy magnetic field is unknown. It is known only that it is formed due to the merging of thin magnetic tubes which is connected to the fact that the density decreases

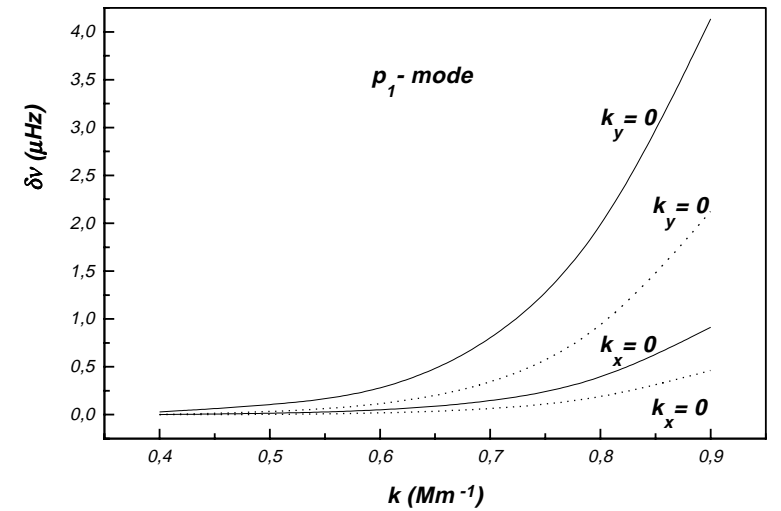

Fig. 3. Shift of the frequencies due to the resonant absorption for $p_{1}$-mode

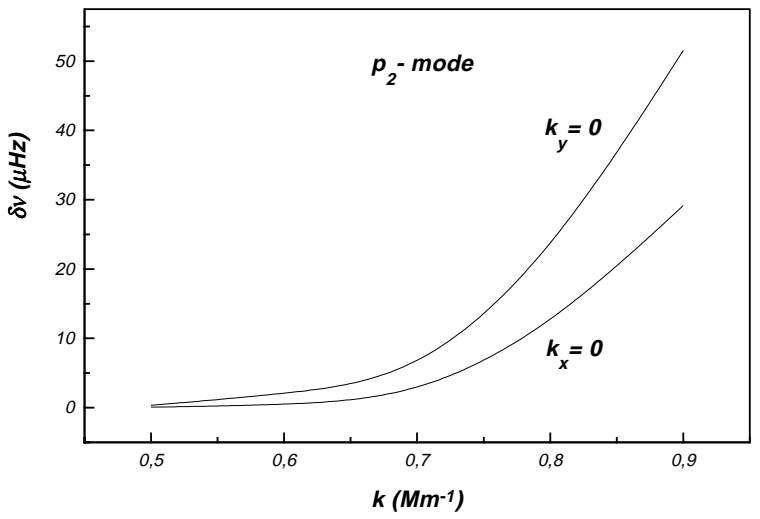

Fig. 4. Shift of the frequencies due to the resonant absorption for $p_{2}$-mode

with height in the chomosphere. According to a rough estimate (Spruit 1981) in quiet areas of the Sun it occurs at the height of $\sim 1600 \mathrm{~km}$.

Thus, to simplify the problem, we assumed here that the canopy magnetic field has the following structure

$H_{0}^{2}(z)=8 H_{\mathrm{m}}^{2} \frac{\exp \left(z / z_{\mathrm{H}}\right)-1}{\left(1+\exp \left(z / z_{\mathrm{H}}\right)\right)^{2}}$

and is determined only by two parameters, $z_{\mathrm{H}}$ and $H_{\mathrm{m}}$.

We found that the inhomogeneity of the magnetic field imposes limitations on the maximum value of the field which can exist in the atmosphere. Figure 1 presents the density variation as a function of height. One can see that for $H_{\mathrm{m}}=28 \mathrm{G}$ is not far from $z / z_{2} \approx 4.4$, the density decreases dramatically almost to zero and as the calculations show, already for $H_{\mathrm{m}} \approx 29 \mathrm{G}$ it becomes negative. Unlike the atmosphere with a homogeneous magnetic field (solid line in Fig. 1), in that with a magnetic field inhomogeneous in height the density displays a local minimum for $z \approx 4.4 z_{2}$ and starts to decrease exponentially only for $z>20 z_{2}$. Therefore, in our study, calculating the spectrum of 5-min oscillations, we adopted $H_{\mathrm{m}}=10 \mathrm{G}$ (in the study by Zhukov 2000 the calculations were performed for the atmosphere with homogeneous magnetic field $H_{0}=30 \mathrm{G}$, i.e. for the value intermediate between 


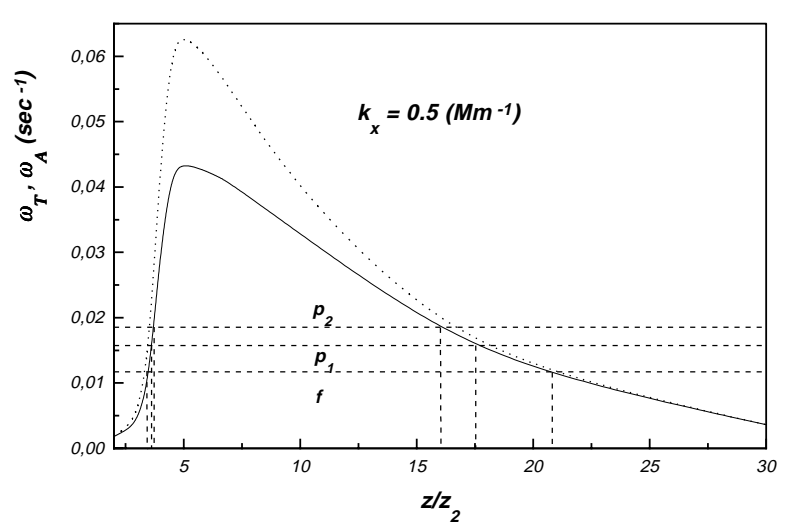

Fig. 5. Cusp (solid line) and Alfvén (dashed line) frequency variation with height for $z_{\mathrm{H}}=2000 \mathrm{~km}$ and $H_{\mathrm{m}}=10 \mathrm{G}$

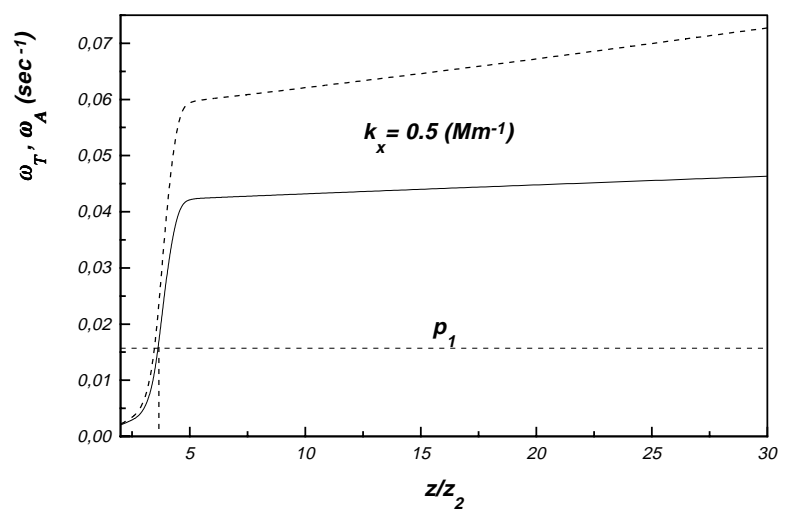

Fig. 6. Cusp (solid line) and Alfvén (dashed line) frequency variation with height for the homogeneous magnetic field $H_{0}=10 \mathrm{G}$

the canopy magnetic field in quiescent and active regions) and $z_{\mathrm{H}}=2000 \mathrm{~km}$ (for this value of $z_{\mathrm{H}}$ the magnetic field reaches its maximum at $\sim 2200 \mathrm{~km}$ ).

\section{Results of calculations}

Zhukov (2000) showed that taking into account the resonant absorption, the spectrum of 5 -min oscillations is continuous. On the diagnostic diagram the eigenfrequencies of 5-min oscillations fill the entire regions limited with the following two curves. One curve represents the eigenfrequencies calculated for $k_{y}=0$, the other for $k_{x}=0$. Therefore here we restricted our consideration to the calculations of only these two curves for $f$-, $p_{1}$ - and $p_{2}$-modes.

The results of the calculations are presented in Figs. 2-4. The shift of eigenfrequencies is denoted by $\delta \nu=\left(\omega_{r}-\omega_{0}\right) / 2 \pi$, where $\omega_{r}$ is the real part of the eigenfrequency for the shell with the magnetic field $H_{0}$, and $\omega_{0}$ is the eigenfrequency for the shell without a magnetic field.

A new, and in our opinion rather important, feature caused by inhomogeneity of the magnetic field is that unlike the atmosphere with a homogeneous magnetic field, in the considered case there are two areas of resonant absorption (see Fig. 5). One is located in the transition region while the other in the lower corona.

In Fig. 5, horizontal dotted straight lines correspond to eigenfrequencies of 5 -min oscillations for $k_{y}=0$ and for $f$-, $p_{1}$ - and $p_{2}$-modes; vertical dotted straight lines determine positions for cusp resonances in the atmosphere. When Fig. 5 is compared with the corresponding Fig. 6 for the atmosphere with a homogeneous magnetic field, it is apparent that, despite the essential coincidence of the position for cusp and Alfvén resonances for $z<5 z_{2}$, the frequency shift is appreciably larger in the atmosphere with an inhomogeneous magnetic field (in Fig. 3 dashed lines indicate the frequency shift for the atmosphere with the homogeneous magnetic field $H_{0}=10 \mathrm{G}$ ). This is apparently due to the presence of the second region of cusp and Alfvén resonances in the lower corona (for $z>15 z_{2}$ ). Thereby, in general, the frequency shift for p-modes can give an insight into the vertical structure of the canopy magnetic field.

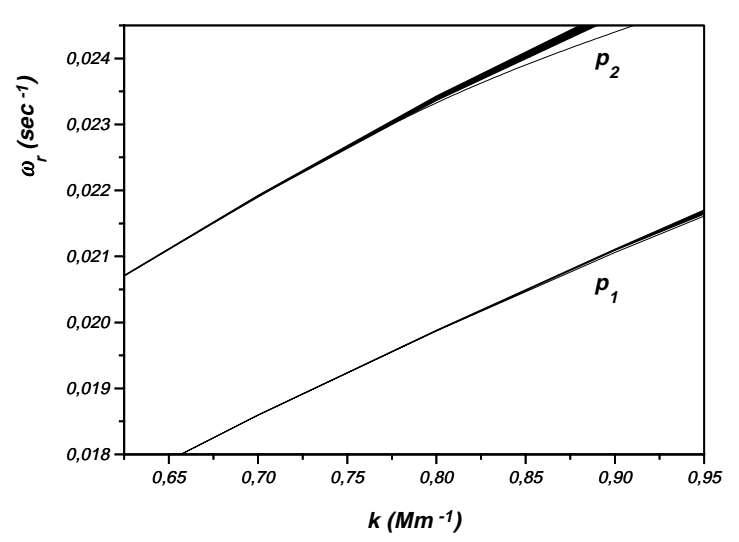

Fig. 7. Diagnostic diagram for 5-min oscillations taking into account their resonant absorption in the region of the canopy magnetic field (solid lines present the diagnostic diagram for the shell without magnetic field)

\section{Conclusions}

Our results show that in the presence of resonant absorption the spectrum of oscillations is continuous, since $k$ can have any value greater than zero. Figure 7 presents the spectrum of 5-min oscillations; shaded areas (which, in fact, represent what we call the fine structure) are limited by two curves, $\omega_{r}(k)$ calculated for $k_{y}=0$ and $k_{x}=0$ (see Figs. 2-4). These areas are the specific features of $k-\omega_{r}$ diagram for 5-min oscillations, taking into account their resonant absorption in the region of the canopy magnetic field. The diagnostic diagram in Fig. 7 displays all basic features: firstly, being narrow for minor $k$, the ridges become broader when $k$ increases, and secondly, the larger the $n$, the larger is the width of the ridges for a given $k$ (compare, for example, Fig. $3(n=1)$ with Fig. $4(n=2))$. As was noted by Zhukov (2000), some indications of the presence of the fine structure in the spectrum of 5-min oscillations shown in Fig. 7 were received by Espagenet et al. (1996), who note that in the domain of very high degree modes, the ridges in the diagnostic diagram should not be as sharp as for the low and medium degree modes. 
Acknowledgements. I would like to thank the referee Dr. S. Hasan for useful suggestions that helped improve the manuscript.

\section{Appendix A}

Taking into account that the eigenfrequencies of oscillations are complex $\left(\omega=\omega_{r}+i \omega_{i}\right)$, the system of Eq. (5) yield the following dispersion relation:

$$
\begin{aligned}
& F_{1}\left(v_{r 1} v_{r 2}-v_{i 1} v_{i 2}\right)-F_{2}\left(v_{r 1} v_{i 2}+v_{r 2} v_{i 1}\right) \\
& -C\left(v_{r 1} \phi_{2}-v_{i 1} \psi_{2}\right)+D\left(v_{r 1} \psi_{2}+v_{i 1} \phi_{2}\right) \\
& +G_{1}\left(v_{r 2} \phi_{1}-v_{i 2} \psi_{1}\right)-G_{2}\left(v_{i 2} \phi_{1}+v_{r 2} \psi_{1}\right)=0 \\
& F_{2}\left(v_{r 1} v_{r 2}-v_{i 1} v_{i 2}\right)+F_{1}\left(v_{r 1} v_{i 2}+v_{r 2} v_{i 1}\right) \\
& -D\left(v_{r 1} \phi_{2}-v_{i 1} \psi_{2}\right)-C\left(v_{r 1} \psi_{2}+v_{i 1} \phi_{2}\right) \\
& +G_{2}\left(v_{r 2} \phi_{1}-v_{i 2} \psi_{1}\right)+G_{1}\left(v_{i 2} \phi_{1}+v_{r 2} \psi_{1}\right)=0 .
\end{aligned}
$$

In the dispersion relation (A.1)

$v_{z n}=v_{r n}+i v_{i n}$,

$\phi_{n}=\frac{\mathrm{d} v_{r n}}{\mathrm{~d} z}, \psi_{n}=\frac{\mathrm{d} v_{i n}}{\mathrm{~d} z}, n=1,2$

where $v_{z n}$ is the vertical component of velocity for the bottom and the top layer correspondingly.

As was already noted, we restricted our study by calculating eigenfrequencies of oscillations only for $k_{y}=0$ and $k_{x}=0$, since for $k_{y} \neq 0, k_{x} \neq 0$ the eigenfrequencies lie within the area limited by two curves, one of which represent the eigenfrequencies calculated for $k_{y}=0$, while the other for $k_{x}=0$.

For $k_{y}=0$, coefficients of the system of Eq. (A.1) are

$$
\begin{aligned}
& F_{1}=g k_{x}\left(S_{r}-1\right)+\frac{\Omega^{2}-c_{02}^{2} k_{x}^{2}}{\rho_{02} c_{02}^{2}} \frac{\mathrm{d}}{\mathrm{d} z}\left(\frac{H_{0}^{2}}{8 \pi}\right), \\
& F_{2}=g k_{x} S_{i}+\frac{W}{\rho_{02} c_{02}^{2}} \frac{\mathrm{d}}{\mathrm{d} z}\left(\frac{H_{0}^{2}}{8 \pi}\right), \\
& C=-\Omega^{2} \\
& D=-W \\
& G_{1}=W S_{i}-\Omega^{2} S_{r}, \\
& G_{2}=-\Omega^{2} S_{i}-W S_{r},
\end{aligned}
$$

where

$S_{r}=\frac{\left(\Omega^{2}-c_{01}^{2} k_{x}^{2}\right)\left(\Omega^{2}-c_{02}^{2} k_{x}^{2}\right)+W^{2}}{\left(\Omega^{2}-c_{01}^{2} k_{x}^{2}\right)^{2}+W^{2}}$,

$S_{i}=W \frac{\left(c_{02}^{2}-c_{01}^{2}\right) k_{x}^{2}}{\left(\Omega^{2}-c_{01}^{2} k_{x}^{2}\right)^{2}+W^{2}}$,

and

$\Omega^{2}=\omega_{r}^{2}-\omega_{i}^{2}, W=2 \omega_{r} \omega_{i}$.

For $k_{x}=0$, coefficients of the system of Eq. (A.1) are

$F_{1}=\frac{g k_{y}^{4}\left(c_{02}^{2}-c_{01}^{2}\right)}{\Omega^{2}-c_{01}^{2} k_{y}^{2}}-\frac{\Omega^{2}}{\rho_{02} c_{02}^{2}} \frac{\mathrm{d}}{\mathrm{d} z}\left(\frac{H_{0}^{2}}{8 \pi}\right)$,

$F_{2}=0$,

$C=\Omega^{2}$,

$D=0$,

$G_{1}=\frac{\Omega^{2}\left(\Omega^{2}-c_{02}^{2} k_{y}^{2}\right)}{\Omega^{2}-c_{01}^{2} k_{y}^{2}}$,

$G_{2}=0$.

\section{References}

Campbell, W. R., \& Roberts, B. 1989, ApJ, 338, 538

Espagenet, O., Muller, R., Roudier, Th., et al. 1996, A\&A, 313, 297

Evans, D. J., \& Roberts, B. 1990, ApJ, 356, 704

Goossens, M. 1991, in Advances in solar system magnetohydrodynamic, ed. E. R. Priest, \& A. W. Hood (Cambridge University Press), 137

Jain, R., \& Roberts, B. 1994a, A\&A, 286, 243

Jain, R., \& Roberts, B. 1994b, A\&A, 286, 254

Nye, A. H., \& Thomas, J. H. 1976, ApJ, 204, 582

Pintér, B., \& Goossens, M. 1999, A\&A, 347, 321

Spruit, H. C. 1981, in The Sun as the Star, ed. S. Jordan, NASA SP $-450,385$

Tirry, W. J., Goossens, M., Pintér, B., Čadež, V., \& Vanlommel, P. 1998, ApJ, 503, 422

Vanlommel, P., \& Goossens, M. 1999, Solar Phys., 187, 357

Zhukov, V. I. 1992, Solar Phys., 139, 201

Zhukov, V. I. 1997a, A\&A, 322, 302, Paper I

Zhukov, V. I. 1997b, Solar Phys., 173, 15

Zhukov, V. I. 2000, A\&A, 354, 277, Paper II 